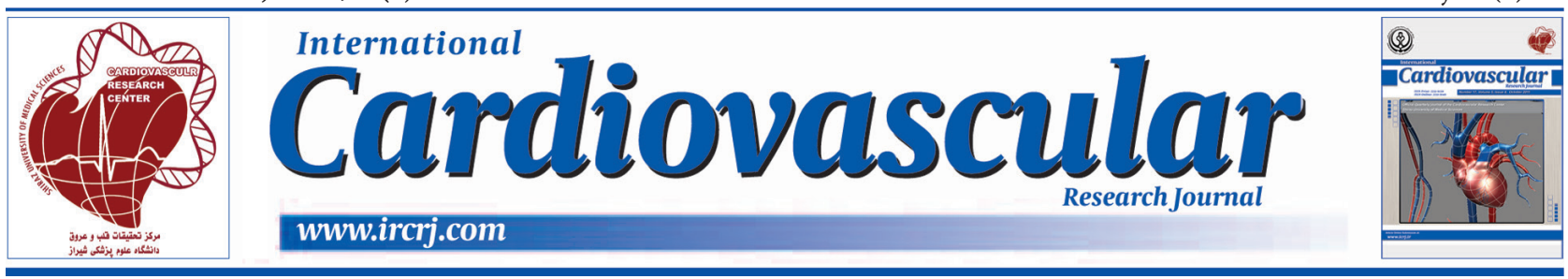

\title{
Pathology of Coronary Chronic Total Occlusion
}

\author{
Kenichi Sakakura ${ }^{1}$, Kazuyuki Yahagi ${ }^{1}$, Renu Virmani ${ }^{1}$, Michael Joner ${ }^{1, *}$
}

${ }^{1}$ CVPath Institute, Gaithersburg, USA

\begin{tabular}{|c|c|}
\hline A R T I C L E I N F O & A B S T R A C T \\
\hline Article Type: & \multirow{10}{*}{$\begin{array}{l}\text { There is an increasing need for percutaneous revascularization procedures of coronary } \\
\text { Chronic Total Occlusion (CTO), because many patients with severe coronary artery } \\
\text { disease have limited options for revascularization. Although the success rate of } \\
\text { percutaneous revascularization of CTOs was unsatisfactory from the 1990s to the 2000s, } \\
\text { recent technological advances in interventional strategies have improved the success } \\
\text { rate to } 85 \% \text {. Detailed histological assessment of human autopsy studies of CTO has } \\
\text { contributed significantly to the refinement in Percutaneous Coronary Intervention (PCI) } \\
\text { techniques and device development. We have recently reported the pathological findings } \\
\text { and characteristics of CTOs that occur in different clinical scenarios. In this review, we } \\
\text { discuss the pathology of CTOs to facilitate greater understanding of revascularization } \\
\text { strategies for CTOs. }\end{array}$} \\
\hline Review Article & \\
\hline Article History: & \\
\hline Received: 24 Jun 2015 & \\
\hline Accepted: 12 Oct 2015 & \\
\hline & \\
\hline Keywords: & \\
\hline Occlusion & \\
\hline Pathology & \\
\hline Percutaneous Coronary Intervention & \\
\hline
\end{tabular}

Implication for health policy/practice/research/medical education:

We have recently reported the pathological findings and characteristics of coronary chronic total occlusions that occur in different clinical scenarios, such as short-duration, long-duration, and post-CABG chronic total occlusion. In this review, we discussed the pathology of chronic total occlusion to facilitate greater understanding of revascularization strategies for coronary chronic total occlusion.

\section{Introduction}

There is growing need for percutaneous revascularization of coronary Chronic Total Occlusion (CTO) as clinical studies have had a beneficial impact on patient outcome following revascularization $(1,2)$. The British Cardiovascular Intervention Society reported that successful CTO Percutaneous Coronary Intervention (PCI) of at least $1 \mathrm{CTO}$ was significantly associated with improved survival (hazard ratio: $0.72 ; 95 \%$ confidence interval: 0.62 to $0.83 ; \mathrm{P}<0.001$ ) compared to unsuccessful CTO (1). Furthermore, although the success rates of percutaneous revascularization of CTOs were not satisfactory, as they ranged from 51 to $74 \%$ up to 2009 (3), recent technological advances and interventional strategies have improved the success rate to $85 \%$ (4-6).

On the other hand, human autopsy studies of CTO have contributed significantly to the refinement of PCI techniques and device developments. Presence of microchannel from recanalization of CTOs described

*Corresponding author: Michael Joner, CVPath Institute, Inc. 19 Firstfield Road, Gaithersburg, MD 20878n, Gaithersburg, USA. Tel: +1-3012083570, Fax: +1-3012083745,

E-mail:mjoner@cvpath.org in human pathological studies $(7,8)$ encouraged the development of smaller diameter guidewires (9). Additionally, the concept of "Loose tissue tracking" was developed from a more fundamental understanding of CTO histopathology (10). Recently, our group reported the pathological definition and characteristics of various types of CTOs (11). In this review, we discuss the pathology of CTOs to further facilitate a greater understanding of revascularization strategies.

\section{Pathological Definition of CTO}

Conflicting data have been reported when comparing angiographic and histopathological CTO definitions. Histopathological assessment of angiographically determined CTO was found frequently to be nonocclusive (8). We have refined the histological definition of CTO (Figure 1) (11) by proposing that the lumen area be occupied by proteoglycan and/or collagen with or without neovascularization and chronic inflammation from the resolved thrombus (11). Furthermore, de novo CTOs were classified into long- and short-duration categories. Long-duration CTO consisted predominantly of matrix composed of type I collagen (type I) in the 
CTOlesion
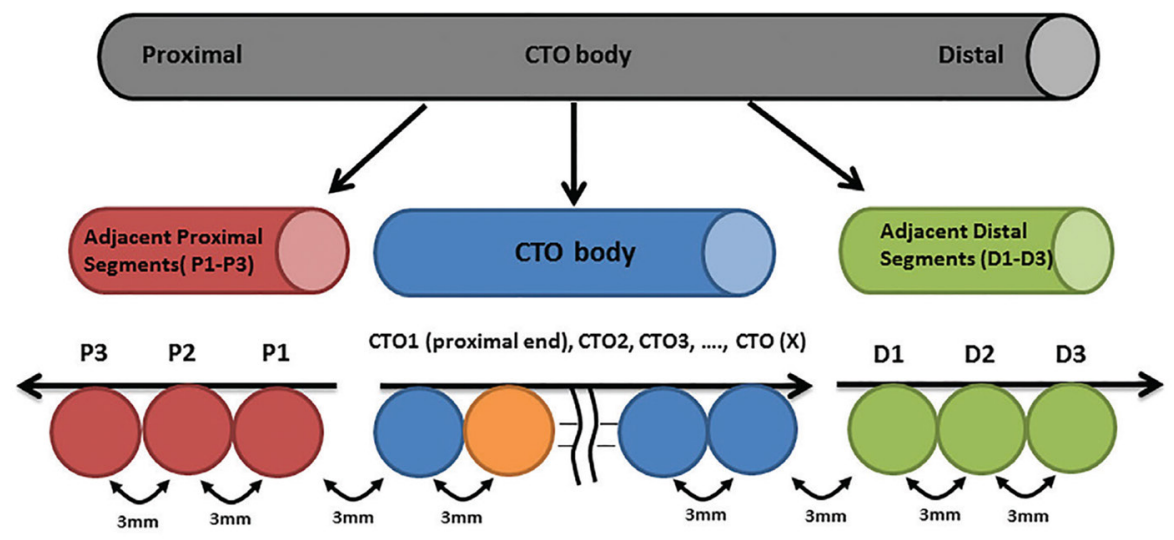

Adjacent Proximal segments. $\leq 95 \%$ stenosis.

Adjacent Distal component. $\leq 95 \%$.

CTO component. At least one total occlusion (>99\% stenosis) or two sections had to have $95 \%>$ stenosis.

Recanalized component. $\leq 95 \%$ stenosis.

Figure 1. The Diagram Illustrates Definition of Chronic Total Occlusion Together with Adjacent Proximal and Distal Segments Studies. Reproduced with Permission from Sakakura et al. (11)

absence of fibrin in all sections of CTO, with negative remodeling (11). Short-duration CTO, on the other hand, was predominantly composed of proteoglycan with or without recanalization and inflammation, but fibrin had to be identified in at least one of the mid-sections (Figure 2) (11).

Figure 2. Representative Images of Long-Duration Chronic Total Occlusion and Short-Duration Chronic Total Occlusion without Coronary Artery Bypass Graft

\section{Components of CTO}

CTO of different durations showed substantial variability in the underlying plaque morphology, such that shortduration CTO showed an organized or organizing thrombus and presence of necrotic core, whereas the predominant component of long-duration CTO was fibrous tissue (11).

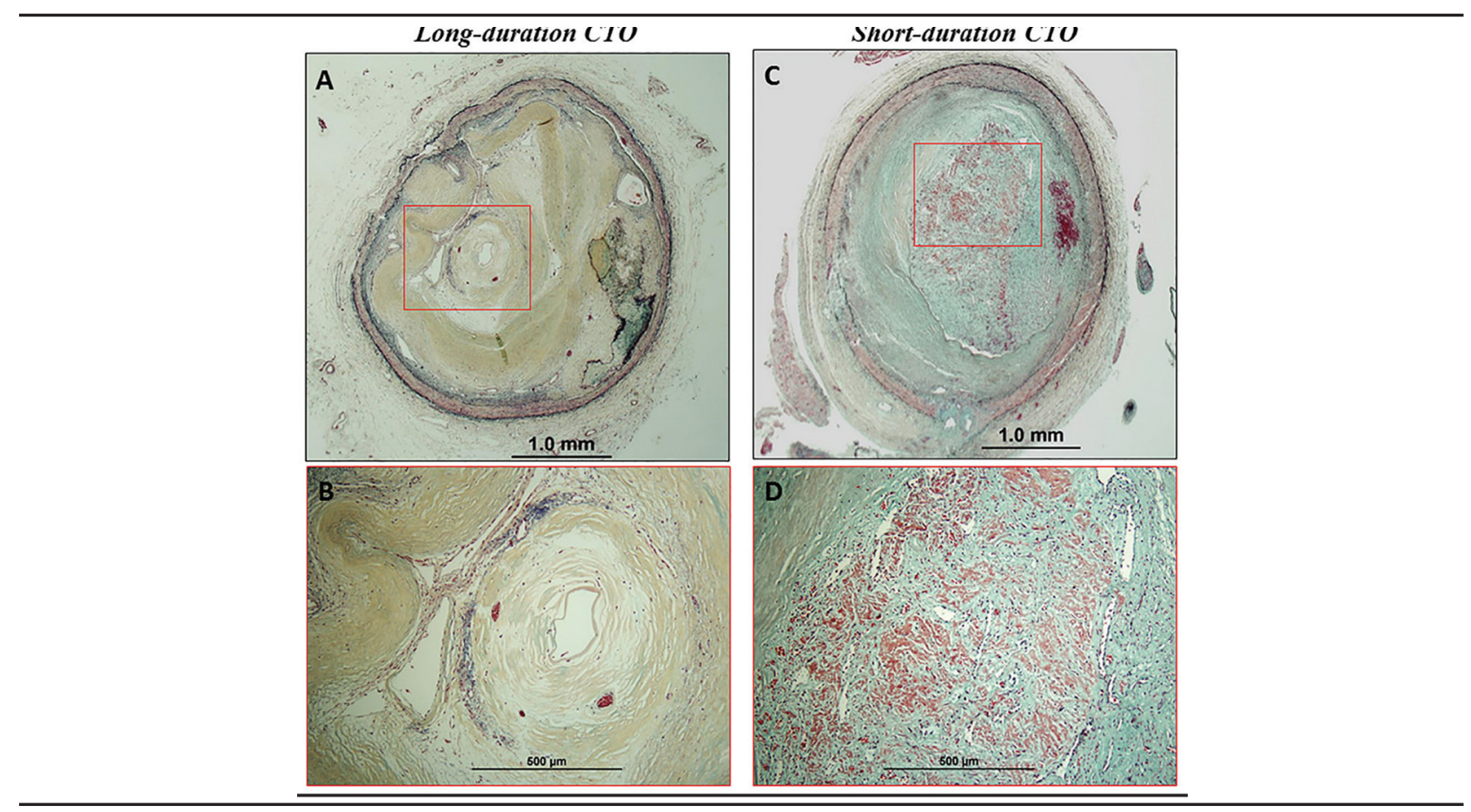

(A and C) Low-power images of long-duration and short-duration chronic total occlusion without coronary artery bypass graft. (B and D) High-power images of boxed areas in (A) and (C), respectively. The matrix is predominantly made up of collagen type I in (B). In (D), the matrix predominantly consists of proteoglycan and fibrin. CTO, chronic total occlusion. Reproduced with permission from Sakakura et al. (11). 
Microchannels consist of small recanalization or thrombi with micro-capillaries. Indeed, approximately $50 \%$ of angiographically determined CTOs show recanalization (8). Katsuragawa et al. reported that the size of small microchannels at autopsy were $160-230 \mu \mathrm{m}$, when present (7), and were observed in 4 out of 10 CTOs (7). Microchannels were only observed in CTOs, which had a tapered proximal lumen pattern, whereas they were absent in CTOs, which had an abrupt proximal lumen pattern (7). In contrast, we reported that small recanalized channels of $>200 \mu \mathrm{m}$ were infrequent among different histological CTOs (11). One potential explanation for this discrepancy is because autopsy studies were initially performed on CTOs that were defined by angiography, which also included incomplete occlusions. Indeed, one quarter of lesions reported by Srivatsa et al. were histologically sub-occlusive (90-95\% stenosis) (8), which is not in agreement with our current definition of CTO. We used a much stricter definition of CTO; i.e., at least one section of CTO (cut at $3 \mathrm{~mm}$ intervals) had to have a total occlusion (>99\% stenosis) or 2 sections with $>95 \%$ stenosis. Nevertheless, microchannels are likely an important guide for the future device improvement, such as small-diameter guidewires or dedicated catheters aimed at crossing CTO lesions. However, it is unlikely that successful recanalization of CTO lesions is based on effective wire passage of microchannels in the range of $20-40 \mu \mathrm{m}$, but rather on targeted penetration of soft tissue, which is composed of proteoglycans, type III collagen, and fibrin within the body of the CTO. Furthermore, micro-capillaries in CTO lesions may connect to the adventitia rather than penetrate through the CTO, but this is not frequent (8). Inflammation also plays an important role in the development of CTO, where densities of macrophages are generally higher in occlusion of $<1$-year duration compared to $>1$-year duration (Figure 3) (12). Lymphocytes and macrophages play an active role in angiogenesis as well as atherosclerotic lesion progression by producing various mitogenic and angiogenic factors that influence the extent of recanalization $(13,14)$.

\section{Negative Remodeling}

One of the major differences between acute thrombotic occlusion and longer duration CTO is the extent of vascular remodeling. Acute thrombi from plaque rupture are associated with positive vascular remodeling (15). Based on animal studies, it is clear that organization of an acute occlusive thrombus leads eventually to vessel remodeling from organization consisting of inflammation, angiogenesis, and Smooth Muscle Cell (SMC) infiltration. SMCs lay down of proteoglycan matrix and collagen type III and I. The type I collagen cross-links with resulting shrinkage of the vessel; i.e., negative remodeling (16). Long-duration CTOs are composed predominantly of type I collagen and exhibit negative remodeling, while short-duration CTOs are composed mostly of MCs, proteoglycan, and fibrin in the absence of negative remodeling (11). The relatively high failure rate of recanalization procedures of long-duration CTOs can be explained on the basis of extensive negative

Figure 3. Role of Inflammation in Formation of Neoangiogenesis in CTO

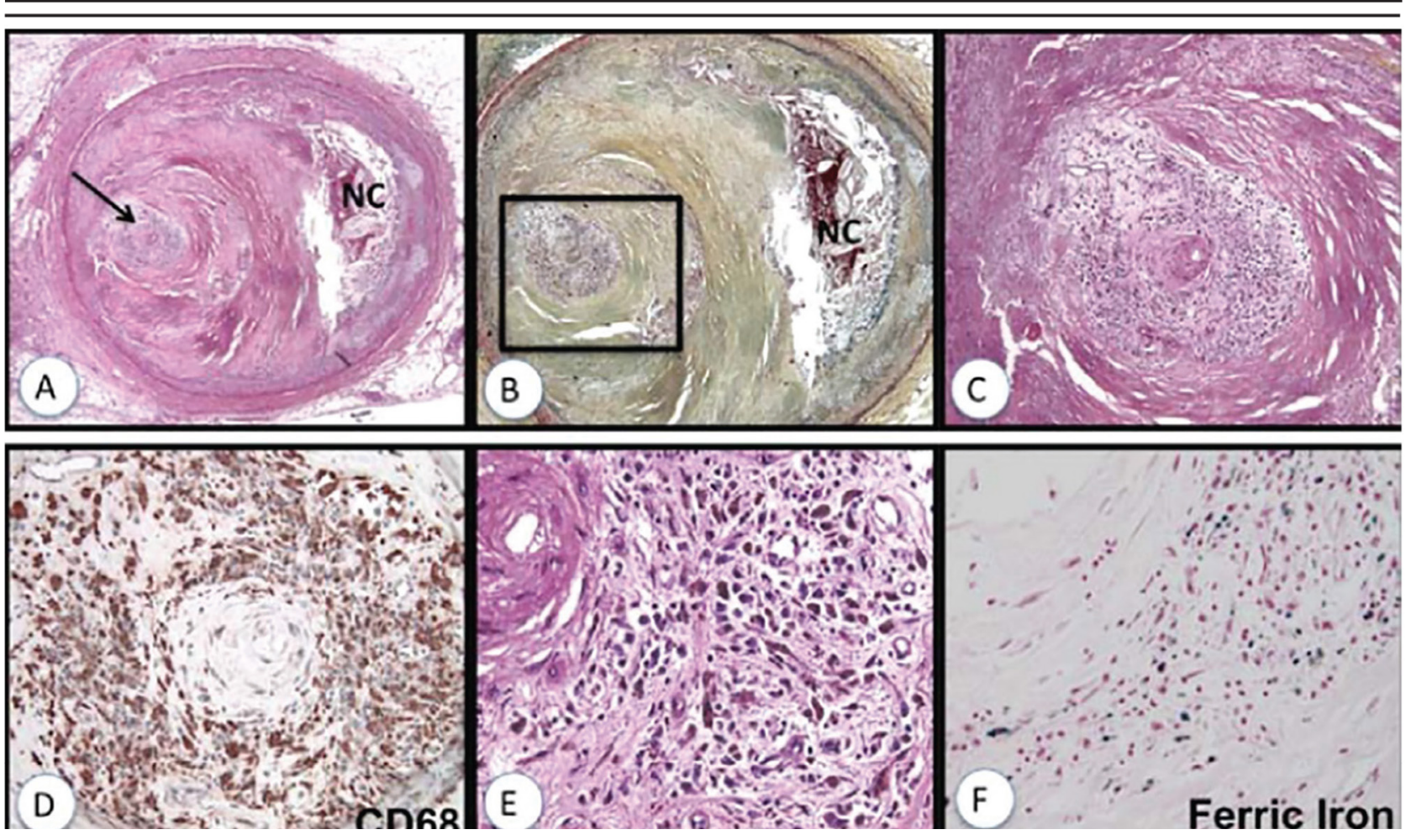

(A) The coronary section of a fibroatheromatous plaque with a Necrotic Core (NC) shows CTO where the lumen is obstructed by a thrombus (arrow), hematoxylin, and eosin stain. (B) Similar sections as in (A) are shown, stained by Movat pentachrome. (C) A higher power view of the area within the black box in (B) shows a thrombus with extensive inflammation and neoangiogenesis. (D) Numerous CD68-positive macrophages are seen in the central lumen. (E) The majority of macrophages within the thrombus contain hemosiderin (brown pigment), hematoxylin, and eosin stain. (F) Pearl's iron stain reveals iron-positive macrophages (blue). Reproduced with permission from Finn et al. (12) 
remodeling and is the likely source for failure of guidewire penetration (17). Morphologic characteristics of CTO have been shown in Figure 4 (12).

\section{Proximal and Distal Lumen Pattern}

It is well known that proximal lumen pattern (tapered or abrupt) is strongly associated with successful antegrade revascularization of CTO $(18,19)$. Tapered lumen pattern is associated with successful guidewire penetration, whereas abrupt lumen pattern with failure of successful wire passage $(18,19)$. In contrast, distal lumen pattern is difficult to judge owing to the difficulty in assessing distal lumen morphology during angiography. We reported that the prevalence of tapered pattern was significantly more frequent in distal lumen compared to proximal lumen (11). The utilization of retrograde approach is one of the main advances in revascularization techniques during CTO procedures and is responsible for the incremental success rates $(6,20)$.

\section{CTO with Coronary Artery Bypass Graft (CABG)}

PCI procedures of $\mathrm{CTO}$ following long-term $\mathrm{CABG}$ are associated with a significantly higher rate of unsuccessful revascularization $(21,22)$. It is reported that approximately half of the patients with prior CABG have native coronary CTO (23). Although conduits in combination with the internal mammary artery have partly replaced the use of saphenous veins during CABG procedures, graft patency rates remain overall unsatisfactory (24). Moreover, shortand long-term outcomes of PCI in saphenous vein graft are poor with drug-eluting stents as well as bare-metal stents $(25,26)$. Therefore, the need to revascularize native coronary CTO with prior CABG is high. We recently reported the pathological characteristics of CTO with prior $\mathrm{CABG}$, in which we showed that severe calcification was a common finding in CTO segments as well as in the proximal and distal adjacent segments (11) (Figure 5). The high failure rate of revascularization attempts in these highly calcified CTO coronary arteries (19) is likely explained by the difficulty encountered during passing of guide wires, even by highly experienced operators. In fact, calcification of CTO segments has been reported to be a significant determinant of unsuccessful CTO PCI patients with prior $\mathrm{CABG}$, and $\mathrm{CTO}$ represents a cohort at high risk for adverse cardiovascular outcomes.

\section{Summary}

Since prior histopathological studies were based on angiographically defined CTO, more accurate histological definition of CTO is an important step for determining better predictors of success and failure following PCI. Histologically, CTO lumen area, if occluded by proteoglycan and/or collagen with or without neovascularization, and chronic inflammation, with the luminal stenosis greater than $95 \%$, have greater chances of PCI success when there is no negative remodeling and there is tapering of proximal non-CTO segments. On the other hand, in long-duration CTO, defined as having a matrix predominately composed of type I collagen and absence of fibrin identified in any histological section of CTO with negative remodeling, the success rates are less and retrograde approach is more likely to be successful. Severely calcified CTO, which

Figure 4. Morphologic Characteristics of CTO
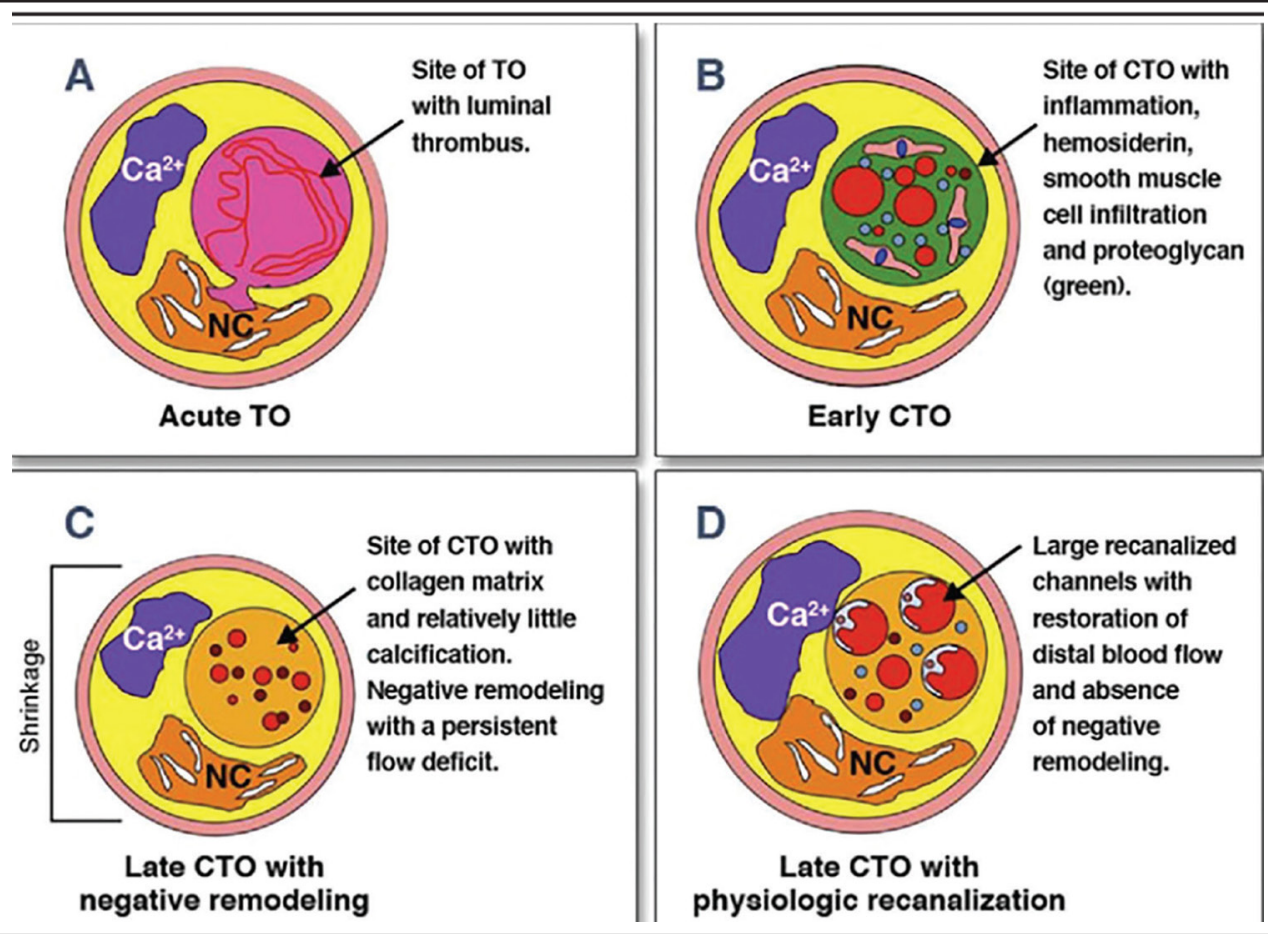

(A) Acute plaque rupture is shown, with luminal thrombus resulting in CTO. (B) Inflammation with early thrombus organization is shown. Note early recanalization accompanied by proteoglycan matrix (green) in the area of Total Occlusion (TO). (C) The late chronic phase of healed TO is shown, with deposition of collagen type I where the cross-linking of collagen promotes negative remodeling of the vessel. (D) Physiological recanalization is accompanied by restoration of normal flow distally, thus preventing negative remodeling. Reproduced with permission from Finn et al. (12) 
Figure 5. A Representative Case of CTO with Coronary Artery Bypass Graft

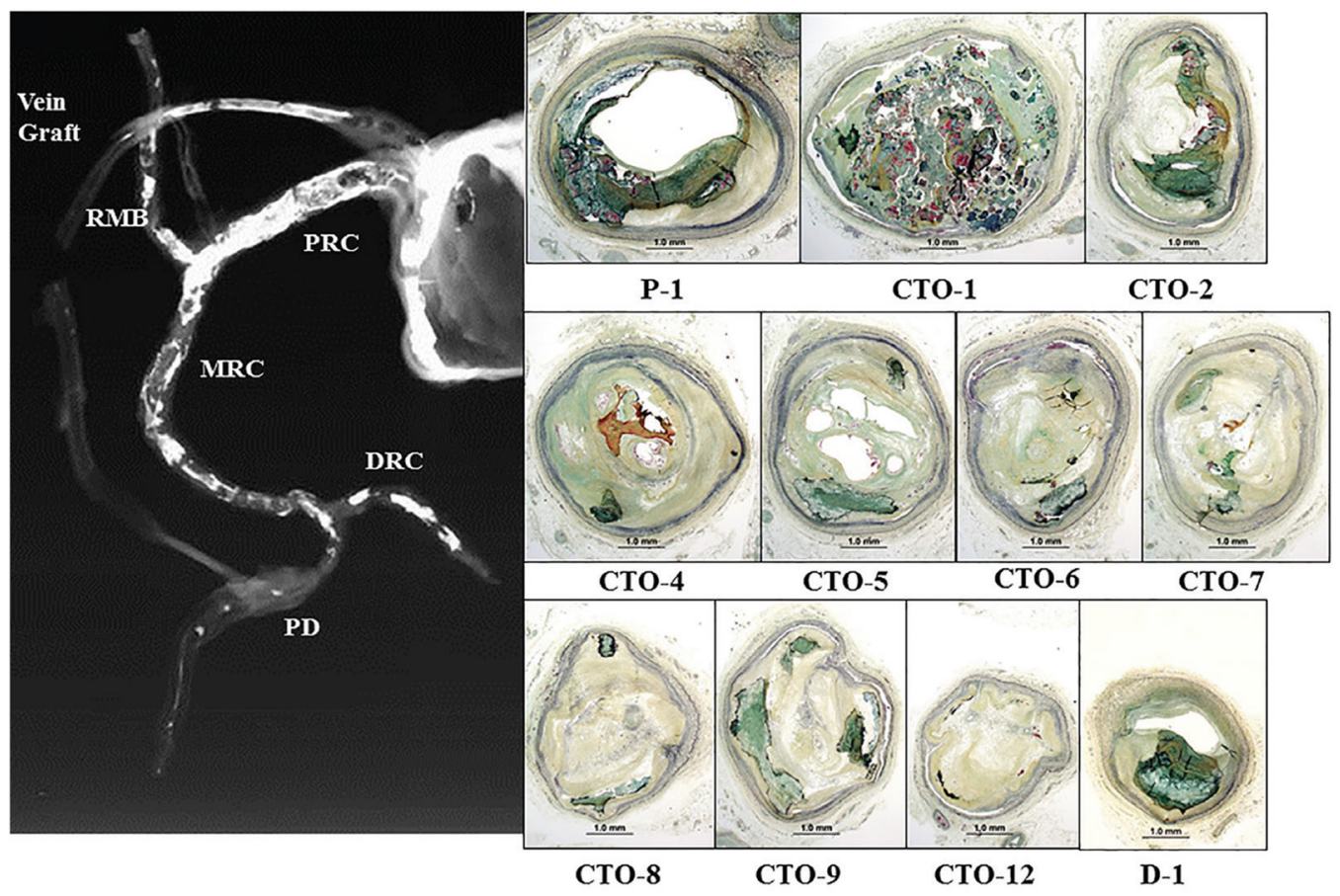

The radiograph (left) shows severe calcification of the vein graft as well as a right coronary artery. P-1 is a proximal segment and D-1 is the distal segment. Serial CTO images show severe calcification. Note: The rate of area calcification in CTO-1 is 70\% and CTO-5 shows five channels $>200 \mathrm{I} 1 / 4 \mathrm{~m}$ in size. The proximal and distal sections to CTO show $54 \%$ and $48 \%$ area calcification, respectively. $\mathrm{PRC}$, proximal right coronary artery; MRC, middle right coronary artery; RMB, right marginal branch; DRC, distal right coronary artery; PD, posterior descending artery; CTO, chronic total occlusion; P-1, proximal first section; D-1, distal first section. Reproduced with permission from Sakakura et al. (11).

is of common occurrence in the native CTO vessels in individuals with long-term CABG, usually does not show negative remodeling but has a proximal abrupt pattern and a distal tapering lumen has a greater success by a retrograde approach during PCI.

\section{Acknowledgments}

The authors thank all members at CVPath Institute Inc., Gaithersburg, MD.

\section{Authors' Contribution}

Kenichi Sakakura drafted the manuscript. Kazuyuki Yahagi, Renu Virmani, and Michael Joner did critical revision of the manuscript for important intellectual content. Renu Virmani and Michael Joner supervised manuscript writing

\section{Financial Disclosure}

There is no sponsor, except for our institution (CVPath Institute, Inc).

\section{Funding/Support}

This work was supported by CVPath Institute, Inc., a private nonprofit research organization.

Kenichi Sakakura received speaking honorarium from Abbott Vascular, Boston Scientific, Daiichi-Sankyo, Medtronic Cardiovascular, Sanofi, and Terumo. Renu Virmani receives research support from Abbott Vascular, BioSensors International, Biotronik, Boston Scientific, Medtronic, MicroPort Medical, Orbus Neich Medical,
SINO Medical Technology, and Terumo Corporation, has speaking engagements with Merck, receives honoraria from Abbott Vascular, Boston Scientific, Lutonix, Medtronic, and Terumo Corporation, and is a consultant for 480 Biomedical, Abbott Vascular, Medtronic, and W.L. Gore. Michael Joner is a consultant for Biotronik and Cardionovum, and has received speaking honorarium from Abbott Vascular, Biotronik, Medtronic, and St. Jude

\section{References}

1. George S, Cockburn J, Clayton TC, Ludman P, Cotton J, Spratt J, et al. Long-term follow-up of elective chronic total coronary occlusion angioplasty: analysis from the U.K. Central Cardiac Audit Database. J Am Coll Cardiol. 2014;64(3):235-43.

2. Olivari Z, Rubartelli P, Piscione F, Ettori F, Fontanelli A, Salemme $\mathrm{L}$, et al. Immediate results and one-year clinical outcome after percutaneous coronary interventions in chronic total occlusions: data from a multicenter, prospective, observational study (TOASTGISE). J Am Coll Cardiol. 2003;41(10):1672-8.

3. Joyal D, Afilalo J, Rinfret S. Effectiveness of recanalization of chronic total occlusions: a systematic review and meta-analysis. Am Heart J. 2010;160(1):179-87.

4. Fang $\mathrm{HY}$, Lee CH, Fang CY, Lin CJ, Wu CC, Yang CH, et al. Application of penetration device (Tornus) for percutaneous coronary intervention in balloon uncrossable chronic total occlusionprocedure outcomes, complications, and predictors of device success. Catheter Cardiovasc Interv. 2011;78(3):356-62.

5. Mitsudo K, Yamashita T, Asakura Y, Muramatsu T, Doi O, Shibata $\mathrm{Y}$, et al. Recanalization strategy for chronic total occlusions with tapered and stiff-tip guidewire. The results of CTO new techniQUE for STandard procedure (CONQUEST) trial. J Invasive Cardiol. 2008;20(11):571-7.

6. Rathore S, Katoh O, Tuschikane E, Oida A, Suzuki T, Takase S. A novel modification of the retrograde approach for the recanalization of chronic total occlusion of the coronary arteries intravascular 
ultrasound-guided reverse controlled antegrade and retrograde tracking. JACC Cardiovasc Interv. 2010;3(2):155-64.

7. Katsuragawa M, Fujiwara H, Miyamae M, Sasayama S. Histologic studies in percutaneous transluminal coronary angioplasty for chronic total occlusion: comparison of tapering and abrupt types of occlusion and short and long occluded segments. J Am Coll Cardiol. 1993;21(3):604-11.

8. Srivatsa SS, Edwards WD, Boos CM, Grill DE, Sangiorgi GM, Garratt KN, et al. Histologic correlates of angiographic chronic total coronary artery occlusions: influence of occlusion duration on neovascular channel patterns and intimal plaque composition. J Am Coll Cardiol. 1997;29(5):955-63.

9. Matsukage T, Masutani M, Yoshimachi F, Takahashi A, Katsuki T, Saito S, et al. A prospective multicenter registry of 0.010 -inch guidewire and compatible system for chronic total occlusion: the PIKACHU registry. Catheter Cardiovasc Interv. 2010;75(7):1006-12.

10. Sumitsuji S, Inoue K, Ochiai M, Tsuchikane E, Ikeno F. Fundamental wire technique and current standard strategy of percutaneous intervention for chronic total occlusion with histopathological insights. JACC Cardiovasc Interv. 2011;4(9):941-51.

11. Sakakura K, Nakano M, Otsuka F, Yahagi K, Kutys R, Ladich E, et al. Comparison of pathology of chronic total occlusion with and without coronary artery bypass graft. Eur Heart J. 2014;35(25):1683-93.

12. Finn AV, Kolodgie FD, Nakano M, Virmani R. The differences between neovascularization of chronic total occlusion and intraplaque angiogenesis. JACC Cardiovasc Imaging. 2010;3(8):806-10.

13. Blotnick S, Peoples GE, Freeman MR, Eberlein TJ, Klagsbrun M. T lymphocytes synthesize and export heparin-binding epidermal growth factor-like growth factor and basic fibroblast growth factor, mitogens for vascular cells and fibroblasts: differential production and release by CD4+ and CD8+ T cells. Proc Natl Acad Sci U S A. 1994;91(8):2890-94.

14. Kumamoto M, Nakashima Y, Sueishi K. Intimal neovascularization in human coronary atherosclerosis: its origin and pathophysiological significance. Hum Pathol. 1995;26(4):450-6.

15. Burke AP, Kolodgie FD, Farb A, Weber D, Virmani R. Morphological predictors of arterial remodeling in coronary atherosclerosis. Circulation. 2002;105(3):297-303.

16. Jaffe R, Leung G, Munce NR, Thind AS, Leong-Poi H, Anderson $\mathrm{KJ}$, et al. Natural history of experimental arterial chronic total occlusions. J Am Coll Cardiol. 2009;53(13):1148-58.

17. Ehara M, Terashima M, Kawai M, Matsushita S, Tsuchikane E, Kinoshita Y, et al. Impact of multislice computed tomography to estimate difficulty in wire crossing in percutaneous coronary intervention for chronic total occlusion. J Invasive Cardiol. 2009;21(11):575-82.

18. Dong S, Smorgick Y, Nahir M, Lotan C, Mosseri M, Nassar H, et al. Predictors for successful angioplasty of chronic totally occluded coronary arteries. J Interv Cardiol. 2005;18(1):1-7.

19. Morino Y, Abe M, Morimoto T, Kimura T, Hayashi Y, Muramatsu T, et al. Predicting successful guidewire crossing through chronic total occlusion of native coronary lesions within 30 minutes: the J-CTO (Multicenter CTO Registry in Japan) score as a difficulty grading and time assessment tool. JACC Cardiovasc Interv. 2011;4(2):213-21.

20. Surmely JF, Katoh O, Tsuchikane E, Nasu K, Suzuki T. Coronary septal collaterals as an access for the retrograde approach in the percutaneous treatment of coronary chronic total occlusions. Catheter Cardiovasc Interv. 2007;69(6):826-32.

21. Michael TT, Karmpaliotis D, Brilakis ES, Abdullah SM, Kirkland $\mathrm{BL}$, Mishoe KL, et al. Impact of prior coronary artery bypass graft surgery on chronic total occlusion revascularisation: insights from a multicentre US registry. Heart. 2013;99(20):1515-8.

22. Michael TT, Karmpaliotis D, Brilakis ES, Fuh E, Patel VG, Mogabgab $\mathrm{O}$, et al. Procedural outcomes of revascularization of chronic total occlusion of native coronary arteries (from a multicenter United States registry). Am J Cardiol. 2013;112(4):488-92.

23. Fefer P, Knudtson ML, Cheema AN, Galbraith PD, Osherov AB, Yalonetsky S, et al. Current perspectives on coronary chronic total occlusions: the Canadian Multicenter Chronic Total Occlusions Registry. J Am Coll Cardiol. 2012;59(11):991-7.

24. Cao C, Ang SC, Wolak K, Peeceeyen S, Bannon P, Yan TD. A metaanalysis of randomized controlled trials on mid-term angiographic outcomes for radial artery versus saphenous vein in coronary artery bypass graft surgery. Ann Cardiothorac Surg. 2013;2(4):401-7.

25. Stone GW, Goldberg S, O'Shaughnessy C, Midei M, Siegel RM, Cristea E, et al. 5-year follow-up of polytetrafluoroethylene-covered stents compared with bare-metal stents in aortocoronary saphenous vein grafts the randomized BARRICADE (barrier approach to restenosis: restrict intima to curtail adverse events) trial. $J A C C$ Cardiovasc Interv. 2011;4(3):300-9.

26. Vermeersch P, Agostoni P, Verheye S, Van den Heuvel P, Convens $\mathrm{C}$, Van den Branden F, et al. Increased late mortality after sirolimuseluting stents versus bare-metal stents in diseased saphenous vein grafts: results from the randomized DELAYED RRISC Trial. $J$ Am Coll Cardiol. 2007;50(3):261-7. 